\title{
QUASI-FUCHSIAN 3-MANIFOLDS AND METRICS ON TEICHMÜLLER SPACE*
}

\author{
REN GUO ${ }^{\dagger}$, ZHENG HUANG ${ }^{\ddagger}$, AND BIAO WANG§
}

\begin{abstract}
An almost Fuchsian 3-manifold is a quasi-Fuchsian manifold which contains an incompressible closed minimal surface with principal curvatures in the range of $(-1,1)$. By the work of Uhlenbeck, such a 3-manifold $M$ admits a foliation of parallel surfaces, whose locus in Teichmüller space is represented as a path $\gamma$, we show that $\gamma$ joins the conformal structures of the two components of the conformal boundary of $M$. Moreover, we obtain an upper bound for the Teichmüller distance between any two points on $\gamma$, in particular, the Teichmüller distance between the two components of the conformal boundary of $M$, in terms of the principal curvatures of the minimal surface in $M$. We also establish a new potential for the Weil-Petersson metric on Teichmüller space.
\end{abstract}

Key words. Quasi-Fuchsian manifolds, foliation, minimal surfaces, Teichmüller distance, WeilPetersson metric.

AMS subject classifications. Primary 30F60, 32G15; Secondary 53C42, 57M50

1. Introduction of Main Results. One of the fundamental questions in hyperbolic geometry of two and three dimensions is the interaction between the internal geometry of a hyperbolic 3-manifold and the geometry of Teichmüller space of Riemann surfaces. It is natural to consider the situation for complete quasi-Fuchsian 3manifolds. Let $M$ be such a manifold, then $M$ is topologically identified as $M=\Sigma \times \mathbb{R}$, where $\Sigma$ is a closed surface of genus $g \geq 2$. We denote the Teichmüller space of genus $g$ surfaces by $\mathcal{T}_{g}(\Sigma)$, the space of conformal structures on $\Sigma$ modulo orientation preserving diffeomorphisms in the homotopy class of the identity map. An important theorem of Brock ([Bro03]), proving a conjecture of Thurston, states that the hyperbolic volume of the convex core of a quasi-Fuchsian 3-manifold is quasi-isometric to the Weil-Petersson distance between the two components of the conformal boundary of the 3-manifold in Teichmüller space.

The space of quasi-Fuchsian 3-manifolds $Q F(\Sigma)$, called the quasi-Fuchsian space, is a complex manifold of complex dimension $6 g-6$. Its geometrical structures are extremely complicated, and they have been subjects of intensive study in recent years. In this paper, we consider mostly a subspace formed by the so-called almost Fuchsian 3-manifolds: a quasi-Fuchsian 3-manifold $M$ is almost Fuchsian if it contains a unique embedded minimal surface, representing the fundamental group, whose principal curvatures are in the range of $(-1,1)$. This subspace is an open connected manifold of the same dimension ([Uhl83]). One can view the quasi-Fuchsian space as a "higher" Teichmüller space, a square with Teichmüller space sitting inside as a diagonal, and view the space of almost Fuchsian 3-manifolds as an open subspace of $Q F(\Sigma)$ around this diagonal. See also $([\mathrm{KS} 07])$ for generalization of almost Fuchsian manifolds to dS, AdS geometry and surfaces with cone points.

By a remarkable theorem of Uhlenbeck ([Uhl83]), any almost Fuchsian 3-manifold admits a foliation of parallel surfaces from the unique minimal surface. These parallel

\footnotetext{
*Received October 3, 2009; accepted for publication March 17, 2010.

${ }^{\dagger}$ School of Mathematics, University of Minnesota, Minneappolis, MN 55455, USA (guoxx170@ math.umn.edu).

${ }^{\ddagger}$ Department of Mathematics, The City University of New York, Staten Island, NY 10314, USA (zheng.huang@csi.cuny.edu).

$\S$ Department of Mathematics, University of Toledo, Toledo, OH 43606, USA (biao.wang@utoledo. edu).
} 
surfaces, denoted by $S(r)$ for $r \in \mathbb{R}$, can be viewed as level sets of distant $r$ from the minimal surface (see $\S 2.2$ for details). Special hypersurfaces such as constant mean curvature surfaces are investigated in [Wan08] and [HW09] and others. The existence of a foliation of submanifolds is an important and powerful tool in the study of geometry. A foliation of parallel surfaces for an almost Fuchsian 3-manifold allows one to relate the deformation of these structures to Teichmüller theory, the deformation theory of conformal structures on a closed surface. We consider a foliation $\mathcal{F}=\{S(r)\}_{r \in \mathbb{R}}$ of parallel surfaces (or the normal flow) from the minimal surface $S$ for an almost Fuchsian 3-manifold $M$ and we are interested in following the locus of this foliation in Teichmüller space: $\{S(r)\}_{r \in \mathbb{R}}$ of $M$ forms a path $\gamma(M)$ in $\mathcal{T}_{g}(\Sigma)$.

THEOREM 1.1. The path $\gamma(M)$ in $\mathcal{T}_{g}(\Sigma)$ joins the conformal structures of the two components of the conformal boundary of $M$.

Starting with any embedded surface with principal curvature in the range of $(-1,1)$ in a quasi-Fuchsian manifold, the foliation of parallel surfaces from this surface forms a path in Teichmüller space. It also joins the two components of conformal boundary. We establish this general situation in the proof, which is based on Esptein's construction in an unpublished manuscript ([Eps84]).

Minsky ([Min93]) considered the locus in Teichmüller space formed by pleated surfaces, and discovered that, in $\epsilon_{0}$-thick part of Teichmüller space, this locus is a quasi-geodesic in the Teichmüller metric.

In this paper, we answer a question raised by Rubstein, where he asked to relate the geometry of an almost Fuchsian 3-manifold to distances in Teichmüller space ([Rub07]). We relate this to the Teichmüller metric and the Weil-Petersson metric.

We obtain an upper bound of the Teichmüller distance $d_{T}$ between the loci of the minimal surface $S$ and $S(r)$ for $r \in(-\infty, \infty)$. In particular, we obtain an upper bound of the Teichmüller distance $d_{T}$ between the conformal structures of fibers $S\left(r_{1}\right)$ and $S\left(r_{2}\right)$ for $r_{1}, r_{2} \in(-\infty, \infty)$.

Theorem 1.2. Let $\lambda_{0}=\max \{\lambda(z), z \in S\}$ be the maximal principal curvature on the minimal surface $S$, then,

$$
\begin{aligned}
d_{T}(S, S(r)) & \leq \frac{1}{2}\left|\log \frac{1+\lambda_{0} \tanh (r)}{1-\lambda_{0} \tanh (r)}\right| \\
d_{T}\left(S\left(r_{1}\right), S\left(r_{2}\right)\right) & \leq \frac{1}{2}\left|\log \frac{1+\lambda_{0} \tanh \left(r_{2}\right)}{1-\lambda_{0} \tanh \left(r_{2}\right)}-\log \frac{1+\lambda_{0} \tanh \left(r_{1}\right)}{1-\lambda_{0} \tanh \left(r_{1}\right)}\right| .
\end{aligned}
$$

A direct consequence is the upper bound for the Teichmüller distance between two conformal boundaries.

Corollary 1.3. If $M=\mathbb{H}^{3} / \Gamma$ is almost Fuchsian with the conformal boundary $\Omega_{+} / \Gamma \sqcup \Omega_{-} / \Gamma$, then

$$
\begin{array}{r}
d_{T}\left(S, \Omega_{ \pm} / \Gamma\right) \leq \frac{1}{2} \log \frac{1+\lambda_{0}}{1-\lambda_{0}} \\
d_{T}\left(\Omega_{-} / \Gamma, \Omega_{+} / \Gamma\right) \leq \log \frac{1+\lambda_{0}}{1-\lambda_{0}} .
\end{array}
$$

One observes that if two conformal boundaries of an almost Fuchsian 3-manifold are far away in Teichmüller metric, then the maximal principal curvature of the unique minimal surface is very close to 1 . 
Epstein ([Eps86]) calculated the maximal dilatation of the hyperbolic Gauss map of a surface homeomorphic to a disk in $\mathbb{H}^{3}$. The inequality (1.4) was essentially due to him ([Eps86] Proposition 5.1). Some local calculations in the proof of (1.2) can also be seen in ([Vel99]).

A rival metric on Teichmüller space to the Teichmüller metric is the WeilPetersson metric, which offers a vastly different view of Teichmüller space: it is incomplete ([Chu76], [Wol75]), Kählerian ([Ahl61]), and with negative sectional curvatures ([Tro86], [Wol86]). We obtain a new potential for the Weil-Petersson metric on Teichmüller space by studying areas of certain immersed surfaces near Fuchsian locus within the quasi-Fuchsian space.

A potential of Weil-Petersson metric is a function defined on Teichmüller space $\mathcal{T}_{g}(\Sigma)$ such that the second variation of this function in direction of two holomorphic quadratic differentials $\alpha d z^{2}$ and $\beta d z^{2}$ at a point of $\mathcal{T}_{g}(\Sigma)$ equals the Weil-Petersson inner product $\langle\alpha, \beta\rangle_{W P}$, up to a constant. Potentials offer important information and computational tools to study the metric. There are several known potentials of the Weil-Petersson metric: Dirichlet energy of harmonic maps ([Tro87], [Wol89], [Jos91]), the Liouville action ([ZT87]), renormalized volume of quasi-Fuchsian manifolds ([TT03], [KS08]) and Hausdorff dimension of the limit set ([BT08], [McM08]). Those potentials are functions well-defined on the whole Teichmüller space. But to calculate the variation, it suffices that a function is defined locally in a neighborhood.

We denote a Riemann surface by $(\Sigma, \sigma)$ where $\sigma$ is a conformal structure. Our new potential of the Weil-Petersson metric is a locally defined function in a neighborhood of $(\Sigma, \sigma) \in \mathcal{T}_{g}(\Sigma)$ which is based on the immersion of a minimal surface in some quasiFuchsian manifold within the quasi-Fuchsian space. The cotangent space of $\mathcal{T}_{g}(\Sigma)$ at a point $(\Sigma, \sigma)$ can be identified with $Q(\sigma)$, the space of holomorphic quadratic differentials on $(\Sigma, \sigma)$. Under this identification, $(\Sigma, \sigma)$ corresponds to the zero quadratic differential denoted by $0 \in Q(\sigma)$. Uhlenbeck ([Uhl83]) showed that there is an open neighborhood $U$ of $0 \in Q(\sigma)$ such that for any holomorphic quadratic differential $\alpha d z^{2} \in U$, there exists a minimal surface $\Sigma\left(\alpha d z^{2}\right)$ immersed in some quasi-Fuchsian 3 -manifold such that the induced metric on $\Sigma\left(\alpha d z^{2}\right)$ is in the conformal class $\sigma$ and the second fundamental form of the immersion is the real part of $\alpha d z^{2}$. The area of $\Sigma\left(\alpha d z^{2}\right)$ with respect to the induced metric, denoted by $\left|\Sigma\left(\alpha d z^{2}\right)\right|$, is a function defined in the neighborhood $U \subset Q(\sigma)$.

THEOREM 1.4. The zero quadratic differential $0 \in Q(\sigma)$ is a critical point of the area functional $\left|\Sigma\left(\alpha d z^{2}\right)\right|: U \rightarrow \mathbb{R}$. And the area functional is a potential of Weil-Petersson metric on Teichmüller space.

Plan of the paper. We provide necessary background material in $\S 2$. The Theorem 1.1, the Theorem 1.2 and the Corollary 1.3 are proved in $\S 3$. In section $\S 4$, the Theorem 1.4 is established.

Acknowledgements. The authors wish to thank Bill Abikoff, Jun $\mathrm{Hu}$, Albert Marden, and Xiaodong Wang for their generous help. We also thank the referee for suggestions to improve this paper. The research of the second named author is partially supported by a PSC-CUNY grant.

2. Preliminaries. The results in this paper lie in the intersection of several different fields, low dimensional topology, geometric function theory and geometric analysis. In this section, we briefly summarize background material for topics involved. We always assume $M$ is orientable and all surfaces involved are closed and orientable of genus at least two. 
2.1. Kleinian groups and quasi-Fuchsian 3-manifolds. A hyperbolic manifold is a complete Riemannian manifold of constant curvature -1 . The universal cover of a hyperbolic manifold is $\mathbb{H}^{n}$, and the deck transformations induce a representation of the fundamental group of the manifold in $\operatorname{Isom}\left(\mathbb{H}^{n}\right)$, the (orientation preserving) isometry group of $\mathbb{H}^{n}$. In the case we are interested in, $\operatorname{Isom}\left(\mathbb{H}^{2}\right)=P S L(2, \mathbb{R})$ which lies naturally in $\operatorname{Isom}\left(\mathbb{H}^{3}\right)=P S L(2, \mathbb{C})$.

A subgroup $\Gamma \subset P S L(2, \mathbb{C})$ is called a Kleinian group if $\Gamma$ acts on $\mathbb{H}^{3}$ properly discontinuously. For any Kleinian group $\Gamma, \forall p \in \mathbb{H}^{3}$, the orbit set

$$
\Gamma(p)=\{\gamma(p) \mid \gamma \in \Gamma\}
$$

has accumulation points on the boundary $S_{\infty}^{2}=\partial \mathbb{H}^{3}$, and these points are the limit points of $\Gamma$, and the closed set of all these points is called the limit set of $\Gamma$, denoted by $\Lambda_{\Gamma}$. The complement of the limit set, i.e.,

$$
\Omega=S_{\infty}^{2} \backslash \Lambda_{\Gamma}
$$

is called the region of discontinuity. $\Gamma$ acts properly discontinuously on $\Omega$, whose quotient $\Omega / \Gamma$ is a finite union of Riemann surfaces of the finite type. The quotient $M=\mathbb{H}^{3} / \Gamma$ is a 3 -manifold with conformal boundary $\Omega / \Gamma$.

Suppose $\Gamma$ is a finitely generated torsion free Kleinian group which has more than two limit points, we call $\Gamma$ quasi-Fuchsian if its limit set $\Lambda_{\Gamma}$ is a closed Jordan curve and both components $\Omega_{ \pm}$of its region of discontinuity are invariant under $\Gamma$. When $\Lambda_{\Gamma}$ is actually a circle, we call $\Gamma$ a Fuchsian group and the corresponding $M$ Fuchsian manifold. It is clear that in this case, $M$ is the product space $\Omega_{+} / \Gamma \times \mathbb{R}$.

If $\Gamma$ is a quasi-Fuchsian group, Marden [Mar74] proved that the quotient $M=$ $\mathbb{H}^{3} / \Gamma$ is diffeomorphic to $\left(\Omega_{+} / \Gamma\right) \times(0,1)$, and $\bar{M}=\left(\mathbb{H}^{3} \cup \Omega\right) / \Gamma$ is diffeomorphic to $\left(\Omega_{+} / \Gamma\right) \times[0,1] . M$ is then called a quasi-Fuchsian 3-manifold.

In this paper we assume that $\Gamma$ contains no parabolic elements, and $M$ is quasiFuchsian but we exclude the case when $M$ is Fuchsian since the locus of the normal flow from a totally geodesic minimal surface is a single point in Teichmüller space. Topologically $M=\Sigma \times \mathbb{R}$ where $\Sigma$ is a closed surface of genus $g \geq 2$. The deformation theory of Kleinian group yields the following simultaneous unformization result, a beautiful theorem of Bers:

TheOREM 2.1. ([Ber60b]) There is a biholomorphic map $Q: \mathcal{T}_{g}(\Sigma) \times \mathcal{T}_{g}(\Sigma) \rightarrow$ $Q F(\Sigma)$ such that $Q(X, Y)$ is the unique quasi-Fuchsian 3-manifold in the quasiFuchsian space $Q F(\Sigma)$ with $X, Y$ as conformal boundaries.

2.2. Foliation of parallel surfaces. In the following we review some results in [Uhl83]. Let $M$ be a quasi-Fuchsian 3-manifold which contains a minimal surface $S$ whose principal curvatures are in the range of $(-1,1)$. Then there is an equidistant foliation of $M$ formed by parallel surfaces $\{S(r)\}_{r \in \mathbb{R}}$. Suppose the coordinate system on $S \equiv S \times\{0\}$ is isothermal so that, using the local coordinate $z=x+i y$, the induced metric $(d x, d y)\left[g_{i j}(z, 0)\right]_{2 \times 2}(d x, d y)^{T}$ on $S$ can be written in the form

$$
\left[g_{i j}(z, 0)\right]=e^{2 v(z)} \mathbb{I}
$$

for some function $v(z)$ and where $\mathbb{I}$ is the $2 \times 2$ identity matrix. Let $(d x, d y)\left[h_{i j}(z, 0)\right]_{2 \times 2}(d x, d y)^{T}$ be the second fundamental form of $S$. We choose $\varepsilon>0$ 
to be sufficiently small, then the (local) diffeomorphism

$$
\begin{aligned}
S \times(-\varepsilon, \varepsilon) & \rightarrow M \\
\left(x^{1}, x^{2}, r\right) & \mapsto \exp _{x}(r \nu)
\end{aligned}
$$

induces a coordinate patch in $M$. Let $S(r)$ be the family of parallel surfaces with respect to $S$, i.e.

$$
S(r)=\left\{\exp _{x}(r \nu) \mid x \in S\right\}, \quad r \in(-\varepsilon, \varepsilon) .
$$

The induced metric $(d x, d y)\left[g_{i j}(z, r)\right](d x, d y)^{T}$ on $S(r)$ can be computed as

$$
\begin{aligned}
{\left[g_{i j}(z, r)\right] } & =\left[g_{i j}(0, r)\right]\left(\cosh r \mathbb{I}+\sinh r\left[g_{i j}(0, r)\right]^{-1}\left[h_{i j}(z, 0)\right]\right)^{2} \\
& =e^{2 v(z)}\left(\cosh r \mathbb{I}+\sinh r e^{-2 v(z)}\left[h_{i j}(z, 0)\right]\right)^{2} .
\end{aligned}
$$

And it is easy to verify, under the principal curvature condition, that induced metric on $S(r)$ (2.1) is well defined for all $r \in \mathbb{R}$. Moreover, the set of parallel surfaces $\{S(r)\}_{r \in \mathbb{R}}$ forms a foliation of $M$, which is called the equidistant foliation or the normal flow.

The second fundamental form $(d x, d y)\left[h_{i j}(z, r)\right](d x, d y)^{T}$ on $S(r)$ is given by

$$
h_{i j}(z, r)=\frac{1}{2} \frac{\partial}{\partial r} g_{i j}(z, r), \quad 1 \leq i, j \leq 2 .
$$

We denote $\lambda(z),-\lambda(z)$ as the principal curvatures of $S$ with $\lambda(z) \geq 0$. Then the principal curvatures of $S(r)$ are given by

$$
\lambda_{1}(z, r)=\frac{\tanh r-\lambda(z)}{1-\lambda(z) \tanh r} \quad \text { and } \quad \lambda_{2}(z, r)=\frac{\tanh r+\lambda(z)}{1+\lambda(z) \tanh r} .
$$

We observe that they are increasing functions of $r$ for fixed $z \in S$, and they both approach \pm 1 when $r \rightarrow \pm \infty$, respectively.

2.3. Metrics on Teichmüller space. For references on quasiconformal mappings and Teichmüller theory, see, for example, the paper [Ber60a] and the book [GL00].

Points in Teichmüller space $\mathcal{T}_{g}(\Sigma)$ are equivalence classes of conformal structures (or alternatively, hyperbolic metrics) on a closed surface $\Sigma$. Two Riemann surfaces $\left(\Sigma, \sigma_{1}\right)$ and $\left(\Sigma, \sigma_{2}\right)$ are equivalent if there is a homeomorphism $f: \Sigma \rightarrow \Sigma$, homotopic to the identity, which is a conformal map from $\left(\Sigma, \sigma_{1}\right)$ to $\left(\Sigma, \sigma_{2}\right)$. Teichmüller space carries a natural topology which is induced by several natural metrics, and among these metrics, the most studied are the Teichmüller metric and the Weil-Petersson metric.

The Teichmüller distance between $\left(\Sigma, \sigma_{1}\right)$ and $\left(\Sigma, \sigma_{2}\right)$ is given by

$$
d_{T}\left(\left(\Sigma, \sigma_{1}\right),\left(\Sigma, \sigma_{2}\right)\right)=\frac{1}{2} \log i n f_{f} K[f],
$$

where $f:\left(\Sigma, \sigma_{1}\right) \rightarrow\left(\Sigma, \sigma_{2}\right)$ is a quasiconformal map homotopic to the identity and $K[f]$ is the maximal dilatation of $f$. This infimum is reached by the Teichmüller map, which yields very geometrical description ([Ber60a]).

The Weil-Petersson metric on Teichmüller space $\mathcal{T}_{g}(\Sigma)$ is induced via an inner product on the cotangent space $Q(\sigma)$. At a point $(\Sigma, \sigma) \in \mathcal{T}_{g}(\Sigma)$, let $\alpha d z^{2}, \beta d z^{2} \in$ 
$Q(\sigma)$ be two holomorphic quadratic differentials on $(\Sigma, \sigma)$. The Weil-Petersson cometric is defined by the Hermitian form

$$
\langle\alpha, \beta\rangle_{W P}=\int_{\Sigma} \frac{\alpha \bar{\beta}}{g_{0}^{2}} d A_{g_{0}}
$$

where $g_{0}|d z|^{2}$ is the unique hyperbolic metric in the conformal class $\sigma$, and $d A_{g_{0}}$ is its area element.

Infinitesimally, the Teichmüller norm of a holomorphic quadratic differential $\phi(z) d z^{2} \in Q(\sigma)$ is the $L^{1}$-norm of $\phi(z) d z^{2}$, while the Weil-Petersson norm is induced by the $L^{2}$-norm.

3. Foliation as a path in Teichmüller space. In this section, we prove the theorems concerning the locus of the foliation $\{S(r)\}_{r \in \mathbb{R}}$ of parallel surfaces for $M$ in Teichmüller space. In $\S 3.1$, we show the Theorem 1.1 and provide alternative point of view from geometric analysis. In $\S 3.2$, we calculate the dilatation of the quasiconformal map from the minimal surface to a fiber $S(r)$, and in $\S 3.3$ we treat the general case, i.e, the quasi-conformal map between any two points on the locus.

3.1. Joining the boundaries. Let $S$ be the minimal surface in an almost Fuchsian manifold $M$, and $\{S(r)\}_{r \in \mathbb{R}}$ be the foliation of parallel surfaces from $S$. The induced metric $(d x, d y)\left[g_{i j}(z, r)\right](d x, d y)^{T}$ on each fiber $S(r)$ is given by $(2.1)$, and such metric determines a conformal structure $\sigma(r)$ on $S(r)$. The path $\gamma$ is formed by the set $\left\{(S(r), \sigma(r)) \in \mathcal{T}_{g}(\Sigma)\right\}_{r \in \mathbb{R}}$. The Theorem 1.1 basically states that the conformal structures determined by the limiting metrics are the conformal boundaries $\Omega_{+} / \Gamma$ and $\Omega_{-} / \Gamma$, prescribed in Bers' Uniformization Theorem 2.1, where $M=\mathbb{H}^{3} / \Gamma$.

Proof of Theorem 1.1. Consider the disjoint decomposition of the boundary of $\mathbb{H}^{3}: S_{\infty}^{2}=\Lambda \sqcup \Omega_{+} \sqcup \Omega_{-}$, where $\Lambda_{\Gamma}$ is the limit set of $\Gamma$, and let $S \subset M$ be an embedded surface (not necessary to be minimal) with principal curvatures in the range of $(-1,1)$. The universal cover of $\left(M, S, \Omega_{+} / \Gamma, \Omega_{-} / \Gamma\right)$ is $\left(\mathbb{H}^{3}, \tilde{S}, \Omega_{+}, \Omega_{-}\right)$, where $\tilde{S}$ is topologically a disk with boundary $\Lambda_{\Gamma}$.

Note that we do not assume $S$ is a minimal surface in this proof, as long as it satisfies the principal curvature condition, or equivalently, the normal flow from $S$ can be extended to infinities. Then $M$ is isometric to $S \times \mathbb{R}$ with the metric

$$
d r^{2}+(d x, d y)\left[g_{i j}(z, r)\right](d x, d y)^{T},
$$

where the second term is the induced metric on $S(r)$ as in (2.1), while $\{z\}$ is the conformal coordinate on $S$.

Let us make it clear how to follow the locus of $S(r)$ in Teichmüller space: for each $r$, the induced metric on $S(r)$ can be expressed in the complex form of $w(z, r) \mid d z+$ $\left.\mu(z, r) d \bar{z}\right|^{2}$. Solving the Beltrami equation $f_{\bar{z}}=\mu(z, r) f_{z}$ for each $r$ provides a quasiconformal map from $S$ to $S(r)$ which is a diffeomorphism a.e., which associates a complex structure on $S(r)$, a point in Teichmüller space ([Ber60a]).

We will work in the universal cover since in what follows is invariant under the action of $\Gamma$. We will use coordinates on $\Omega_{+}$to write the embedding of $\tilde{S}$ into $\mathbb{H}^{3}$. $\mathbb{H}^{3}$ is identified with $B^{3}=\left\{(x, y, z) \mid x^{2}+y^{2}+z^{2}<1\right\}$ and $S_{\infty}^{2}$ with the unit sphere

$$
S^{2}=\left\{X(\theta):=\left(\frac{2 \Re \theta}{|\theta|^{2}+1}, \frac{2 \Im \theta}{|\theta|^{2}+1}, \frac{|\theta|^{2}-1}{|\theta|^{2}+1}\right) \mid \theta \in S_{\infty}^{2}\right\} .
$$


Given a point $X(\theta) \in S^{2}$, there is unique horoshpere $H(\theta)$ at $X(\theta)$ tangent to $\tilde{S}$. Therefore $\tilde{S}$ determines a function $\rho: S^{2} \rightarrow \mathbb{R}$ on $\Omega_{+}$whose absolute value is the hyperbolic distance from $(0,0,0) \in B^{3}$ to $H(\theta)$, and $\rho(\theta)$ is positive when $(0,0,0)$ is out of $H(\theta)$, and it is negative when $(0,0,0)$ is in $H(\theta)$. $B^{3}$ :

Using the function $\rho(\theta)$, Epstein $([$ Eps84] (2.4)) obtained the embedding of $\tilde{S}$ in

$$
\begin{aligned}
\Omega_{+} & \rightarrow B^{3} \\
\theta & \mapsto \frac{|D \rho|^{2}+\left(e^{2 \rho}-1\right)}{|D \rho|^{2}+\left(e^{\rho}+1\right)^{2}} X(\theta)+\frac{2 D \rho}{|D \rho|^{2}+\left(e^{\rho}+1\right)^{2}},
\end{aligned}
$$

where $D \rho$ is the gradient of $\rho(\theta)$ with respect to the canonical metric on $S^{2}: \frac{4|d \theta|^{2}}{\left(1+|\theta|^{2}\right)^{2}}$.

With above formula of the embedding, one can work out explicitly the first and second fundamental forms of $\tilde{S}$

$$
\begin{aligned}
& (d \Re \theta, d \Im \theta)\left[\bar{g}_{i j}(\theta, 0)\right](d \Re \theta, d \Im \theta)^{T}, \\
& (d \Re \theta, d \Im \theta)\left[\bar{h}_{i j}(\theta, 0)\right](d \Re \theta, d \Im \theta)^{T},
\end{aligned}
$$

in the coordinate $\theta$ (see $[\operatorname{Eps} 84](5.4,5.6))$.

As in the formula $(2.1)$, the induced metric $(d \Re \theta, d \Im \theta)\left[\bar{g}_{i j}(\theta, r)\right](d \Re \theta, d \Im \theta)^{T}$ on the parallel surface $\widetilde{S(r)}$, with distance $r>0$ to $\tilde{S}$ is again

$$
\left[\bar{g}_{i j}(\theta, r)\right]=\left[\bar{g}_{i j}(\theta, 0)\right]\left(\cosh r \mathbb{I}+\sinh r\left[\bar{g}_{i j}(\theta, 0)\right]^{-1}\left[\bar{h}_{i j}(\theta, 0)\right]\right)^{2} .
$$

When $r \rightarrow \infty$, the matrix $\left[\bar{g}_{i j}(\theta, r)\right]$ does not converge. Using the matrix $\cosh ^{-2} r\left[\bar{g}_{i j}(\theta, r)\right]$ which induces the same conformal structure as $\left[\bar{g}_{i j}(\theta, r)\right]$, we get the limit (see [Eps84] (5.10)):

$$
\begin{aligned}
\lim _{r \rightarrow \infty} \cosh ^{-2}(r)\left[\bar{g}_{i j}(\theta, r)\right] & =\left[\bar{g}_{i j}(\theta, 0)\right]\left(\mathbb{I}+\left[\bar{g}_{i j}(\theta, 0)\right]^{-1}\left[\bar{h}_{i j}(\theta, 0)\right]\right)^{2} \\
& =e^{2 \rho(\theta)} \frac{4}{\left(1+|\theta|^{2}\right)^{2}} \mathbb{I}
\end{aligned}
$$

We can check that, for any element $\gamma \in \Gamma$, the function $\rho(\theta)$ satisfies

$$
e^{2 \rho(\gamma(\theta))} \frac{\left|\gamma^{\prime}(\theta)\right|^{2}}{\left(1+|\gamma(\theta)|^{2}\right)^{2}}=e^{2 \rho(\theta)} \frac{1}{\left(1+|\theta|^{2}\right)^{2}}
$$

Therefore $e^{2 \rho(\theta)} \frac{4|d \theta|^{2}}{\left(1+|\theta|^{2}\right)^{2}}$ defines a metric on $\Omega_{+} / \Gamma$ which is conformal under the coordinate $\theta$. Thus this metric induces the same conformal structure on $\Omega_{+} / \Gamma$ coming from Bers's Uniformization.

It is clear that one can use above calculation for $r<0$ to treat the case of the Riemann surface $\Omega_{-} / \Gamma$.

We want to also provide an alternative approach to the Theorem 1.1, in a much more general setting. Let $\bar{N}$ be a compact manifold with boundary $\partial N$. We call $f$ a defining function if $f$ is a smooth function on $\bar{N}$ with a first order zero on $\partial N$. A Riemannian metric $g$ on $N=\operatorname{int}(\bar{N})$ is called conformally compact if for any defining function $f, f^{2} g$ extends as a smooth metric on $\bar{N}$, whose restriction defines a metric and a well-defined conformal structure $C$ on $\partial N$. The pair $(\partial N, C)$ is the conformal 
infinity of $N$. A quasi-Fuchsian manifold is a conformally compact Einstein manifold and the conformal infinity of a conformal compact Einstein manifold is independent of the choice of boundary defining functions ([FG85], [GL91]). Therefore we need to find a defining function for the metric $g_{M}$ in formula (3.1).

Let $t=\tanh (r)$, then $t \in(-1,1)$ and the formula (3.1) can be written as

$$
g_{M}^{\prime}=\frac{1}{\left(1-t^{2}\right)^{2}} d t^{2}+\frac{1}{1-t^{2}}(d x, d y) h(z, t)(d x, d y)^{T},
$$

where $h(z, t)=e^{2 v(z)}\left[\mathbb{I}+t e^{-2 v(z)} A(z)\right]^{2}$ and $z=x+i y$. Then $M$ is isometric to $\Sigma \times(-1,1)$ with the metric $g_{M}^{\prime}$.

Now we define $f(t)=1-t^{2}$, then $f( \pm 1)=0$ and $f^{\prime}( \pm 1) \neq 0$, and

$$
f^{2}(t) g_{M}^{\prime}=d t^{2}+\left(1-t^{2}\right)(d x, d y) h(z, t)(d x, d y)^{T} .
$$

It is then easy to see that $f^{2}(t) g_{M}^{\prime}$ is a smooth metric on the compactified $\bar{M}=$ $M \cup \partial M$, isometric to $\Sigma \times[-1,1]$, with conformal infinity the disjoint union of two conformal structures achieved by taking $t= \pm 1$. Therefore by above definitions, $g(z, r)$, hence the parallel surfaces, approach two components of the conformal infinity.

REMARK 3.1. The question whether the locus $\gamma$ is simple in Teichmüller space is not known.

3.2. Teichmüller distance bounds.. In this subsection, we prove formulas (1.1) and (1.3), i.e, we compare the conformal structure on $S(r)$ with the minimal surface $S$. Solutions are in a simpler form when we use conformal coordinates on a minimal surface since the mean curvature is zero.

The strategy is quite straightforward: we express the induced metric $(d x, d y)\left[g_{i j}(z, r)\right](d x, d y)^{T}$ on $S(r)$ in its complex form, then estimate the dilatation for the quasi-conformal map which assigns the complex structure on $S(r)$.

We fix the metric $(d x, d y)\left[g_{i j}(z, 0)\right](d x, d y)^{T}=e^{2 v(z)} d z d \bar{z}$ on $S$ and the second fundamental form $(d x, d y)\left[h_{i j}(z, 0)\right](d x, d y)^{T}$. Since the surface $S$ is minimal, we may write the matrix $e^{-2 v(z)}\left[h_{i j}(z, 0)\right]$ as

$$
\left(\begin{array}{cc}
a & b \\
b & -a
\end{array}\right)
$$

with $\pm \lambda$ as eigenvalues, i.e., the principal curvatures of $S$. And we define $\lambda_{0}>0$ as the maximum of the principal curvatures of $S$. It is easy to see that $a^{2}+b^{2}=\lambda^{2}$.

Let $p^{r}$ be the intersection point of $S(r)(r \in(-\infty, \infty))$ and the geodesic perpendicular to $S$ at $p$. There is a diffeomorphism $u^{r}: S \rightarrow S(r)$ sending $p$ to $p^{r}$.

Lemma 3.2. This map $u^{r}: S \rightarrow S(r)$ is $\lambda_{0}|\tanh (r)|$-quasiconformal.

Proof. Assume $\rho^{2} d \zeta d \bar{\zeta}$ is the induced metric on $S(r)$ from the embedding $S(r) \rightarrow$ $M$, in terms of the conformal coordinate $\zeta$. To calculate the complex dilatation, we need to write the pull-back $\left(u^{r}\right)^{*} \rho^{2} d \zeta d \bar{\zeta}$ in its complex form $w(z, r)|d z+\mu(z, r) d \bar{z}|^{2}$ using the coordinate $z$ on $S$.

Write $z=x+i y, \alpha:=\cosh (r)$ and $\beta:=\sinh (r)$. We find that 


$$
\begin{aligned}
\left(u^{r}\right)^{*} \rho^{2} d \zeta d \bar{\zeta} & =(d x, d y) e^{2 v(z)}\left(\cosh r \mathbb{I}+\sinh r e^{-2 v(z)}\left[h_{i j}(z, 0)\right]\right)^{2}(d x, d y)^{T} \\
& =(d x, d y) e^{2 v(z)}\left(\begin{array}{cc}
\alpha+a \beta & b \beta \\
b \beta & \alpha-a \beta
\end{array}\right)^{2}(d x, d y)^{T} \\
& =: E d x^{2}+2 F d x d y+G d y^{2}
\end{aligned}
$$

where

$$
\begin{aligned}
& E=e^{2 v(z)}\left(\alpha^{2}+2 a \alpha \beta+\beta^{2} \lambda^{2}\right) \\
& F=e^{2 v(z)}(2 b \alpha \beta) \\
& G=e^{2 v(z)}\left(\alpha^{2}-2 a \alpha \beta+\beta^{2} \lambda^{2}\right) .
\end{aligned}
$$

From standard Riemannian geometry, we have

$$
\mu(z, r)=\frac{E-G+2 \sqrt{-1} F}{E+G+2 \sqrt{E G-F^{2}}} .
$$

Here we have

$$
E G-F^{2}=e^{4 v(z)}\left(\alpha^{2}-\lambda^{2} \beta^{2}\right)^{2},
$$

and the complex dilatation of $u^{r}$ in a neighborhood of $p \in S$ is

$$
\mu(z, r)=\tanh (r)(a+\sqrt{-1} b),
$$

with $|\mu(z, r)|=|\lambda(z) \tanh (r)| \leq \lambda_{0}|\tanh (r)|<1$. Since the surface $\Sigma$ is closed, $u^{r}$ is $\lambda_{0}|\tanh (r)|$-quasiconformal.

Directly from (2.3), the Teichmüller distance between $S$ and $S(r)$ is bounded from above, i.e.,

$$
d_{T}(S, S(r)) \leq \frac{1}{2} \log \frac{1+\lambda_{0}|\tanh (r)|}{1-\lambda_{0}|\tanh (r)|}<\frac{1}{2} \log \frac{1+\lambda_{0}}{1-\lambda_{0}} .
$$

This completes the parts (1.1) and (1.3) in the Theorem 1.2, and the Corollary 1.3.

3.3. Teichmüller distance bounds: general case. In this subsection, we complete the proof of the Theorem 1.2. The same strategy applies: given any two fiber surfaces $S\left(r_{1}\right)$ and $S\left(r_{2}\right)$, we determine the quasi-conformal map between them obtained from solving the Beltrami equation, and estimate the Beltrami coefficient.

There is a natural map from $u: S\left(r_{1}\right) \rightarrow S\left(r_{2}\right)$ given by the normal flow. More precisely, let $p \in S\left(r_{1}\right)$. Then $p^{\prime}=u(p)$ is the intersection point of $S\left(r_{2}\right)$ and the geodesic perpendicular to $S\left(r_{1}\right)$ at $p$.

Lemma 3.3. The map $u$ is $k$-quasi-conformal with $k=\frac{\lambda_{0}\left|\tanh \left(r_{2}\right)-\tanh \left(r_{1}\right)\right|}{1-\lambda_{0}^{2} \tanh \left(r_{2}\right) \tanh \left(r_{1}\right)}$.

Proof. Locally the metric on $S\left(r_{1}\right)$ is $e^{2 v^{\prime}} d z^{\prime} d \overline{z^{\prime}}$. The second fundamental form is $\left[h_{i j}^{\prime}\left(z^{\prime}, 0\right)\right]$ and we write the matrix $e^{-2 v^{\prime}\left(z^{\prime}\right)}\left[h_{i j}^{\prime}\left(z^{\prime}, 0\right)\right]$ as

$$
\left(\begin{array}{ll}
a & b \\
b & c
\end{array}\right)
$$


with $\lambda_{j}$ as eigenvalues.

Therefore $a+c=\lambda_{1}+\lambda_{2}$ and $a c-b^{2}=\lambda_{1} \lambda_{2}$.

The pull-back metric on $S\left(r_{2}\right)$ by $u$ is then given by

$$
\begin{aligned}
& \left(d x^{\prime}, d y^{\prime}\right) e^{2 v^{\prime}\left(z^{\prime}\right)}\left(\cosh \left(r_{2}-r_{1}\right) \mathbb{I}+\sinh \left(r_{2}-r_{1}\right) e^{-2 v\left(z^{\prime}\right)}\left[h_{i j}^{\prime}\left(z^{\prime}, 0\right)\right]\right)^{2}\left(d x^{\prime}, d y^{\prime}\right)^{T} \\
= & \left(d x^{\prime}, d y^{\prime}\right) e^{2 v^{\prime}\left(z^{\prime}\right)}\left(\begin{array}{cc}
\alpha+a \beta & b \beta \\
b \beta & \alpha+c \beta
\end{array}\right)^{2}\left(d x^{\prime}, d y^{\prime}\right)^{T} \\
= & : E^{\prime} d x^{2}+2 F^{\prime} d x d y+G^{\prime} d y^{2} .
\end{aligned}
$$

Here we write $\alpha=\cosh \left(r_{2}-r_{1}\right)$ and $\beta=\sinh \left(r_{2}-r_{1}\right)$, and

$$
\begin{aligned}
& E^{\prime}=e^{2 v^{\prime}\left(z^{\prime}\right)}\left(\alpha^{2}+2 a \alpha \beta+\beta^{2}\left(a^{2}+b^{2}\right)\right) \\
& F^{\prime}=e^{2 v^{\prime}\left(z^{\prime}\right)}\left(2 b \alpha \beta+b \beta^{2}(a+c)\right) \\
& G^{\prime}=e^{2 v^{\prime}\left(z^{\prime}\right)}\left(\alpha^{2}+2 c \alpha \beta+\beta^{2}\left(c^{2}+b^{2}\right)\right) .
\end{aligned}
$$

Now the metric $E^{\prime} d x^{2}+2 F^{\prime} d x d y+G^{\prime} d y^{2}$ can be written in the form $w^{\prime}\left(z^{\prime}\right) \mid d z^{\prime}+$ $\left.\mu\left(z^{\prime}\right) d \overline{z^{\prime}}\right|^{2}$. It is easy to verify

$$
E^{\prime} G^{\prime}-F^{\prime 2}=e^{4 v^{\prime}\left(z^{\prime}\right)}\left(\alpha+\lambda_{1} \beta\right)^{2}\left(\alpha+\lambda_{2} \beta\right)^{2},
$$

and

$$
E^{\prime}+G^{\prime}+2 \sqrt{E^{\prime} G^{\prime}-F^{\prime 2}}=e^{2 v^{\prime}\left(z^{\prime}\right)}\left(2 \alpha+\left(\lambda_{1}+\lambda_{2}\right) \beta\right)^{2},
$$

and

$$
E^{\prime}-G^{\prime}+2 \sqrt{-1} F^{\prime}=e^{2 v^{\prime}\left(z^{\prime}\right)} \beta\left(2 \alpha+\left(\lambda_{1}+\lambda_{2}\right) \beta\right)(a-c+2 b \sqrt{-1}) .
$$

Therefore, applying (3.2), we obtain

$$
\begin{aligned}
\mu\left(z^{\prime}\right) & =\frac{E^{\prime}-G^{\prime}+2 \sqrt{-1} F^{\prime}}{E^{\prime}+G^{\prime}+2 \sqrt{E^{\prime} G^{\prime}-F^{\prime 2}}} \\
& =\frac{\beta(a-c+2 \sqrt{-1} b)}{2 \alpha+\left(\lambda_{1}+\lambda_{2}\right) \beta}
\end{aligned}
$$

and

$$
\begin{aligned}
\left|\mu\left(z^{\prime}\right)\right| & =\frac{\beta\left|\lambda_{2}-\lambda_{1}\right|}{\left|2 \alpha+\left(\lambda_{1}+\lambda_{2}\right) \beta\right|} \\
& =\frac{\left|\lambda_{2}-\lambda_{1}\right|}{\left|\lambda_{1}+\lambda_{2}+2 \operatorname{coth}\left(r_{2}-r_{1}\right)\right|} .
\end{aligned}
$$

On the other hand, as in the formula (2.2), the principal curvatures $\lambda_{1}\left(z, r_{1}\right), \lambda_{2}\left(z, r_{1}\right)$ on $S\left(r_{1}\right)$ can be expressed in terms of the principal curvatures of the minimal surface $S: \pm \lambda(z)$. Thus, we find

$$
\left|\mu\left(z^{\prime}\right)\right|=\frac{\lambda(z)\left|\tanh \left(r_{2}\right)-\tanh \left(r_{1}\right)\right|}{1-\lambda^{2}(z) \tanh \left(r_{2}\right) \tanh \left(r_{1}\right)} .
$$

Since $\left|\mu\left(z^{\prime}\right)\right|$ is an increasing function of $\lambda$ for fixed $r_{1}$ and $r_{2}$, we now find

$$
\left|\mu\left(z^{\prime}\right)\right| \leq \frac{\lambda_{0}\left|\tanh \left(r_{2}\right)-\tanh \left(r_{1}\right)\right|}{1-\lambda_{0}^{2} \tanh \left(r_{2}\right) \tanh \left(r_{1}\right)}
$$


where $\lambda_{0}$ again is the maximum of principal curvatures on $S$.

It is now easy to see that

$$
\begin{aligned}
\frac{\lambda_{0}\left|\tanh \left(r_{2}\right)-\tanh \left(r_{1}\right)\right|}{1-\lambda_{0}^{2} \tanh \left(r_{2}\right) \tanh \left(r_{1}\right)} & <\frac{\left|\tanh \left(r_{2}\right)-\tanh \left(r_{1}\right)\right|}{1-\tanh \left(r_{2}\right) \tanh \left(r_{1}\right)} \\
& =\left|\tanh \left(r_{2}-r_{1}\right)\right|<1,
\end{aligned}
$$

and then $u$ is $\frac{\lambda_{0}\left|\tanh \left(r_{2}\right)-\tanh \left(r_{1}\right)\right|}{1-\lambda_{0}^{2} \tanh \left(r_{2}\right) \tanh \left(r_{1}\right)}$-quasiconformal.

The Theorem 1.2 follows easily from the Lemma 3.3 and the Corollary 1.3 is obtained by triangle inequality or taking $r_{1}$ and $r_{2}$ to $-\infty$ and $\infty$.

4. Weil-Petersson potential. In this section, we prove the Theorem 1.4: the induced area functional of minimal surfaces in the quasi-Fuchsian space is a potential at the Fuchsian locus for the Weil-Petersson metric on Teichmüller space. It is natural to consider the hyperbolic metrics on a surface $\Sigma$ rather than the conformal structures in the geometry of the Weil-Petersson metric. A key fact for our theorem is that the second fundamental form of a minimal surface is the real part of a holomorphic quadratic differential.

Let $g_{0}|d z|^{2}$ be the hyperbolic metric on $(\Sigma, \sigma)$, where $\sigma$ is a conformal structure on $\Sigma$. By the Uniformization Theorem, the set of conformal structures and the set of hyperbolic metrics are of one-to-one correspondence. The hyperbolic surface $\Sigma$ with metric $g_{0}|d z|^{2}$ can be immersed into the Fuchsian manifold $\Sigma \times \mathbb{R}$ as a (totally geodesic) minimal surface with the vanishing second fundamental form.

Lemma 4.1. ([Uhl83]) There is an open neighborhood $U$ of $0 \in Q(\sigma)$ such that for any $\alpha d z^{2} \in U$, there exists a minimal surface $\Sigma\left(\alpha d z^{2}\right)$, immersed in some quasiFuchsian manifold such that the induced metric on $\Sigma\left(\alpha d z^{2}\right)$ is $e^{2 u} g_{0}|d z|^{2}$ and the second fundamental form of the immersion is the real part of $\alpha d z^{2}$.

Proof of Theorem 1.4. We start with the classical Gauss equation. Using the fact that curvature of the hyperbolic metric $g_{0}|d z|^{2}$ is -1 , one can rewrite Gauss equation as the following quasi-linear elliptic equation:

$$
\Delta_{g_{0}} u+1-e^{2 u}-\frac{|\alpha|^{2}}{g_{0}^{2}} e^{-2 u}=0,
$$

where $\Delta_{g_{0}}$ is the Laplace operator on the hyperbolic surface $\Sigma$ with metric $g_{0}|d z|^{2}$.

Now consider a one-parameter family of Gauss equations for metrics on a minimal surface in the conformal class of the hyperbolic metric $g_{0}|d z|^{2}$, and second fundamental form $\Re\left(t \alpha d z^{2}\right)$ for a fixed holomorphic quadratic differential $\alpha d z^{2} \in Q(\sigma)$.

For each small $t$, the solution $u^{t}$ to

$$
\Delta_{g_{0}} u^{t}+1-e^{2 u^{t}}-\frac{|t \alpha|^{2}}{g_{0}^{2}} e^{-2 u^{t}}=0 .
$$

gives rise to an immersed minimal surface $\Sigma(t \alpha)$ with principal curvatures in the range of $(-1,1)$.

At $t=0$, the maximum principle implies the solution to

$$
\Delta_{g_{0}} u^{0}=-1+e^{2 u^{0}}
$$

is exactly $u^{0}=0$, hence $u^{0}=0$ corresponds to the totally geodesic case. 
The (induced) area functional of $\Sigma\left(t \alpha d z^{2}\right)$ is given by

$$
\left|\Sigma\left(t \alpha d z^{2}\right)\right|=\int_{\Sigma} e^{2 u^{t}} d A_{g_{0}} .
$$

We denote $\dot{u}=\left.\frac{\partial u}{\partial t}\right|_{t=0}$. Differentiating (4.2) respect to $t$ and evaluate at $t=0$, we get

$$
\Delta_{g_{0}} \dot{u}-2 \dot{u}=0 .
$$

By the maximum principle, we have

$$
\dot{u}=0 .
$$

The first variation of the area is then

$$
\left.\frac{\partial}{\partial t}\right|_{t=0}\left(\left|\Sigma\left(t \alpha d z^{2}\right)\right|\right)=\int_{\Sigma} 2 e^{2 u^{0}} \dot{u} d A_{g_{0}}=0 .
$$

Hence the area functional is critical at the Fuchsian locus.

We now consider the second variation, let $t \alpha d z^{2}+s \beta d z^{2} \in U \subset Q(\sigma)$ with $t, s \in \mathbb{C}$ and $|t|,|s|$ small enough. The area of $\Sigma\left(t \alpha d z^{2}+s \beta d z^{2}\right)$ is

$$
\left|\Sigma\left(t \alpha d z^{2}+s \beta d z^{2}\right)\right|=\int_{\Sigma} e^{2 u(t, s)} d A_{g_{0}},
$$

where $u(t, s)$ satisfies

$$
\Delta_{g_{0}} u(t, s)+1-e^{2 u(t, s)}-\frac{|t \alpha+s \beta|^{2}}{g_{0}^{2}} e^{-2 u(t, s)}=0,
$$

with $u(0,0)=0$.

Differentiating (4.4) respect to $t$ and $\bar{s}$ and evaluating at $t=s=0$, we obtain

$$
\left.\Delta_{g_{0}} \frac{\partial^{2} u}{\partial \bar{s} \partial t}\right|_{t=s=0}-\left.2 \frac{\partial^{2} u}{\partial \bar{s} \partial t}\right|_{t=s=0}-\frac{\alpha \bar{\beta}}{g_{0}^{2}}=0 .
$$

Here we also used (4.3). Integrating the two sides of (4.5), we find

$$
\begin{aligned}
0 & =\left.\int_{\Sigma} \Delta_{g_{0}} \frac{\partial^{2} u}{\partial \bar{s} \partial t}\right|_{t=s=0} d A_{g_{0}} \\
& =\left.\int_{\Sigma} 2 \frac{\partial^{2} u}{\partial \bar{s} \partial t}\right|_{t=s=0} d A_{g_{0}}+\int_{\Sigma} \frac{\alpha \bar{\beta}}{g_{0}^{2}} d A_{g_{0}} .
\end{aligned}
$$

Therefore

$$
\begin{aligned}
& \frac{\partial^{2}}{\partial \bar{s} \partial t}\left|\Sigma\left(t \alpha d z^{2}+s \beta d z^{2}\right)\right|_{t=s=0} \\
= & \left.\left.\int_{\Sigma} 4 \frac{\partial u}{\partial \bar{s}}\right|_{t=s=0} \frac{\partial u}{\partial t}\right|_{t=s=0} d A_{g_{0}}+\left.\int_{\Sigma} 2 \frac{\partial^{2} u}{\partial \bar{s} \partial t}\right|_{t=s=0} d A_{g_{0}} \\
= & -\int_{\Sigma} \frac{\alpha \bar{\beta}}{g_{0}^{2}} d A_{g_{0}},
\end{aligned}
$$

here again we used (4.3). Now the second variation of the area functional is a constant multiple of the Weil-Petersson pairing between holomorphic quadratic differentials $\alpha d z^{2}$ and $\beta d z^{2}$ by (2.4). 


\section{REFERENCES}

[Ahl61] L. V. Ahlfors, Some remarks on Teichmüller's space of Riemann surfaces, Ann. of Math. (2), 74 (1961), pp. 171-191.

[Ber60a] L. Bers, Quasiconformal mappings and Teichmüller's theorem, Analytic functions, Princeton Univ. Press, Princeton, N.J., 1960, pp. 89-119.

[Ber60b] - Simultaneous uniformization, Bull. Amer. Math. Soc., 66 (1960), pp. 94-97.

[Bro03] J. F. Brock, The Weil-Petersson metric and volumes of 3-dimensional hyperbolic convex cores, J. Amer. Math. Soc., 16:3 (2003), pp. 495-535 (electronic).

[BT08] M. J. Bridgeman and E. C. TAYlor, An extension of the Weil-Petersson metric to quasi-Fuchsian space, Math. Ann., 341:4 (2008), pp. 927-943.

[Chu76] T. Chu, The Weil-Petersson metric in the moduli space, Chinese J. Math., 4:2 (1976), pp. 29-51.

[Eps84] C. L. EPSTEIn, Envelopes of horospheres and weingarten surfaces in hyperbolic 3-space, unpublished manuscript.

[Eps86] - The hyperbolic Gauss map and quasiconformal reflections, J. Reine Angew. Math., 372 (1986), pp. 96-135.

[FG85] C. Fefferman and C. R. Graham, Conformal invariants, Astérisque (1985), no. Numero Hors Serie, pp. 95-116, The mathematical heritage of Élie Cartan (Lyon, 1984).

[GL91] C. R. GRAhAm AND J. M. LeE, Einstein metrics with prescribed conformal infinity on the ball, Adv. Math., 87:2 (1991), pp. 186-225.

[GL00] F. P. GARDiner AND N. LAKIC, Quasiconformal Teichmüller theory, Mathematical Surveys and Monographs, vol. 76, American Mathematical Society, Providence, RI, 2000 .

[HW09] Z. HUANG AND B. WANG, Geometric evolution equations and foliations on quasi-fuchsian three-manifolds, preprint (2009).

[Jos91] J. Jost, Two-dimensional geometric variational problems, Pure and Applied Mathematics (New York), John Wiley \& Sons Ltd., Chichester, 1991, A Wiley-Interscience Publication.

[KS07] K. KRASNOv AND J.-M. Schlenker, Minimal surfaces and particles in 3-manifolds, Geom. Dedicata, 126 (2007), pp. 187-254.

[KS08] - On the renormalized volume of hyperbolic 3-manifolds, Com. Math. Phy., 279:3 (2008), pp. 637-668.

[Mar74] A. Marden, The geometry of finitely generated kleinian groups, Ann. of Math. (2), 99 (1974), pp. 383-462.

[McM08] C. T. MCMullen, Thermodynamics, dimension and the Weil-Petersson metric, Invent. Math., 173:2 (2008), pp. 365-425.

[Min93] Y. N. MinsKY, Teichmüller geodesics and ends of hyperbolic 3-manifolds, Topology, 32:3 (1993), pp. 625-647.

[Rub07] J. H. Rubinstein, Problems around 3-manifolds, Workshop on Heegaard Splittings, Geom. Topol. Monogr., vol. 12, Geom. Topol. Publ., Coventry, 2007, pp. 285-298.

[Tro86] A. J. Tromba, On a natural algebraic affine connection on the space of almost complex structures and the curvature of Teichmüller space with respect to its Weil-Petersson metric, Manuscripta Math., 56:4 (1986), pp. 475-497.

[Tro87] - On an energy function for the Weil-Petersson metric on Teichmüller space, Manuscripta Math., 59:2 (1987), pp. 249-260.

[TT03] L. A. TAKhtajan And L.-P. TeO, Liouville action and Weil-Petersson metric on deformation spaces, global Kleinian reciprocity and holography, Comm. Math. Phys., 239:1-2 (2003), pp. 183-240.

[Uhl83] K. K. UhlenBeck, Closed minimal surfaces in hyperbolic 3-manifolds, Seminar on minimal submanifolds, Ann. of Math. Stud., vol. 103, Princeton Univ. Press, Princeton, NJ, 1983, pp. 147-168.

[Vel99] J. A. Velling, Existence and uniqueness of complete constant mean curvature surfaces at infinity of $\mathbf{H}^{3}$, J. Geom. Anal., 9:3 (1999), pp. 457-489.

[Wan08] B. WAng, Foliations for quasi-Fuchsian 3-manifolds, J. Differential Geom., (2008), to appear.

[Wol75] S. A. Wolpert, Noncompleteness of the Weil-Petersson metric for Teichmüller space, Pacific J. Math., 61:2 (1975), pp. 573-577.

[Wol86] - Chern forms and the Riemann tensor for the moduli space of curves, Invent. Math., 85:1 (1986), pp. 119-145.

[Wol89] M. Wolf, The Teichmüller theory of harmonic maps, J. Differential Geom., 29:2 (1989), pp. $449-479$. 
[ZT87] P. G. ZOGRAF AND L. A. TAKHTADZhyAn, On the uniformization of Riemann surfaces and on the Weil-Petersson metric on the Teichmüller and Schottky spaces, Mat. Sb. (N.S.), 132(174):3 (1987), pp. 304-321, 444. 